PROCEEDINGS OF THE

AMERICAN MATHEMATICAL SOCIETY

Volume 134, Number 1, Pages 149-156

S 0002-9939(05)08312-7

Article electronically published on August 19, 2005

\title{
ON REGULARITY CRITERIA IN TERMS OF PRESSURE FOR THE NAVIER-STOKES EQUATIONS IN $\mathbb{R}^{3}$
}

\author{
YONG ZHOU
}

(Communicated by David S. Tartakoff)

\begin{abstract}
In this paper we establish a Serrin-type regularity criterion on the gradient of pressure for the weak solutions to the Navier-Stokes equations in $\mathbb{R}^{3}$. It is proved that if the gradient of pressure belongs to $L^{\alpha, \gamma}$ with $2 / \alpha+3 / \gamma \leq 3,1 \leq \gamma \leq \infty$, then the weak solution is actually regular. Moreover, we give a much simpler proof of the regularity criterion on the pressure, which was showed recently by Berselli and Galdi (Proc. Amer. Math. Soc. 130 (2002), no. 12, 3585-3595).
\end{abstract}

\section{INTRODUCTION}

We consider the following Cauchy problem for the incompressible Navier-Stokes equations in $\mathbb{R}^{3} \times(0, T)$ :

$$
\left\{\begin{array}{l}
\frac{\partial u}{\partial t}+u \cdot \nabla u+\nabla p=\Delta u \\
\operatorname{div} u=0 \\
u(x, 0)=u_{0}(x)
\end{array}\right.
$$

where $u=u(x, t) \in \mathbb{R}^{3}$ is the velocity field, $p(x, t)$ is a scalar pressure, and $u_{0}(x)$ with $\operatorname{div} u_{0}=0$ in the sense of distribution is the initial velocity field.

The study of the incompressible Navier-Stokes equations in three space dimensions has a long history (see [5, 19]). In the pioneering work 12] and [7, Leray and Hopf proved the existence of its weak solutions $u(x, t) \in L^{\infty}\left(0, T ; L^{2}\left(\mathbb{R}^{3}\right)\right) \cap$ $L^{2}\left(0, T ; H^{1}\left(\mathbb{R}^{3}\right)\right)$ for given $u_{0}(x) \in L^{2}\left(\mathbb{R}^{3}\right)$. However, we do not yet know whether or not the solution develops singularities in finite time even if the initial datum is $C^{\infty}$-smooth. In [15], Scheffer began to study the partial regularity theory of the Navier-Stokes equations. Deeper results were obtained by Caffarelli, Kohn and Nirenberg in [3]. Further results can be found in [20] and the references therein.

On the other hand, the regularity of a given weak solution $u$ can be shown under additional conditions. In 1962, Serrin [16] proved that if $u$ is a Leray-Hopf weak solution belonging to $L^{\alpha, \gamma} \equiv L^{\alpha}\left(0, T ; L^{\gamma}\left(\mathbb{R}^{3}\right)\right)$ with $2 / \alpha+3 / \gamma<1,2<$ $\alpha<\infty, 3<\gamma<\infty$, then the solution $u(x, t)$ belongs to $C^{\infty}\left(\mathbb{R}^{3} \times(0, T]\right)$, while the limit case $2 / \alpha+3 / \gamma=1$ was covered much later by H. Sohr [17] (recently,

Received by the editors February 3, 2004.

2000 Mathematics Subject Classification. Primary 35B45, 35B65, 76D05.

Key words and phrases. Navier-Stokes equations, regularity criterion, integrability of pressure, a priori estimates.

(C)2005 American Mathematical Society Reverts to public domain 28 years from publication 
Beirão da Veiga [1] added Serrin's condition on only two components of the velocity field). From then on, there are many criterion results added on $u$. In [21] and [6], von Wahl and Giga showed that if $u$ is a weak solution in $C\left([0, T) ; L^{3}\left(\mathbb{R}^{3}\right)\right)$, then $u(x, t) \in C^{\infty}\left(\mathbb{R}^{3} \times(0, T]\right)$. Struwe [18 proved the same regularity of $u$ in $L^{\infty}\left(0, T ; L^{3}\left(\mathbb{R}^{3}\right)\right.$ provided $\sup _{0<t \leq T}\|u(x, t)\|_{L^{3}}$ is sufficiently small, and Kozono and Sohr [10] obtained the regularity for the weak solution $u(x, t) \in C^{\infty}\left(\mathbb{R}^{3} \times(0, T]\right)$ provided $u(x, t)$ is left continuous with respect to the $L^{3}$-norm for every $t \in(0, T)$. Recently Kozono and Taniuchi 11 showed that if a Leray-Hopf weak solution $u(x, t) \in L^{2}(0, T ; B M O)$, then $u(x, t)$ is actually a strong solution of (1.1) on $(0, T]$. Recent progress concerning another limit case $u \in L^{\infty}\left(0, T ; L^{3}\right)$ can be found in 8

It is well known that if $(u, p)$ solves the Navier-Stokes equations, then so does $\left(u_{\lambda}, p_{\lambda}\right)$ for all $\lambda>0$, where $u_{\lambda}(x, t)=\lambda u\left(\lambda x, \lambda^{2} t\right), p_{\lambda}(x, t)=\lambda^{2} p\left(\lambda x, \lambda^{2} t\right)$. The class of Serrin's type is important from a viewpoint of scaling invariance, which implies that $\left\|u_{\lambda}\right\|_{L^{\alpha, \gamma}}=\|u\|_{L^{\alpha, \gamma}}$ holds for all $\lambda>0$ if and only if $2 / \alpha+3 / \gamma=1$, and we say that the norm $\|u\|_{L^{\alpha, \gamma}}$ has the scaling dimension zero [3].

It is easy to check that if $2 / \alpha+3 / \gamma=3,\|\nabla p\|_{L^{\alpha, \gamma}}$ has scaling dimension zero. As far as we know, there are only few regularity criteria in terms of $\nabla p$; see [2, 14]. The best result [2] for the whole space is that

$$
\nabla p \in L^{\alpha}\left(0, T ; L^{\gamma}\left(\mathbb{R}^{3}\right)\right) \text { with } \frac{2}{\alpha}+\frac{3}{\gamma}=3, \text { for } \gamma \in[9 / 7,3] .
$$

In 2, regularity criteria were established not only for the whole space, but also for a domain with boundary (bounded, exterior or the half-space). The purpose of this paper is to establish a final regularity criterion in terms of the gradient of pressure. Our main theorem reads

Theorem 1.1. Let $u_{0}(x) \in L^{2}\left(\mathbb{R}^{3}\right) \cap L^{q}\left(\mathbb{R}^{3}\right)$, for $q \geq 4$, and let $\operatorname{div} u_{0}=0$ in the sense of distribution. Suppose that $u(x, t)$ is a Leray-Hopf weak solution of (1.1). If

$$
\nabla p \in L^{\alpha}\left(0, T ; L^{\gamma}\left(\mathbb{R}^{3}\right)\right) \text { with } \frac{2}{\alpha}+\frac{3}{\gamma} \leq 3, \frac{2}{3}<\alpha<\infty, 1<\gamma<\infty,
$$

or $\nabla p \in L^{2 / 3, \infty}$, or else $\|\nabla p\|_{L^{\infty, 1}}$ is sufficiently small, then $u(x, t)$ is a regular solution on $[0, T]$.

Remark 1.1. For Navier-Stokes equations in a domain $\Omega \subsetneq \mathbb{R}^{3}$, it is very difficult; cf. [2, 22].

In section 3, we will give a much simpler proof for the following known result. Moreover, our method can treat $\gamma$ uniformly instead of a different trick for different $\gamma$ as done in [2, 4].

Theorem 1.2 ([2]). Under the same assumption as Theorem 1.1, if

$$
p \in L^{\alpha}\left(0, T ; L^{\gamma}\left(\mathbb{R}^{3}\right)\right) \text { with } 2 / \alpha+3 / \gamma \leq 2,1<\alpha<\infty, 3 / 2<\gamma<\infty,
$$

then $u(x, t)$ is a regular solution on $[0, T]$.

Remark 1.2. The limit cases $p \in L^{1, \infty}$ or $\|p\|_{L^{\infty, 3 / 2}}$ being sufficiently small were treated in [4]. 


\section{Proof of Theorem 1.1}

First, we should establish an a priori estimate.

Taking $\nabla$ div on both sides of (1.1) for smooth $(u, p)$, one can obtain

$$
-\Delta(\nabla p)=\sum_{i, j=1}^{3} \partial_{i} \partial_{j}\left(\nabla\left(u_{i} u_{j}\right)\right) .
$$

Therefore the Calderon-Zygmund inequality

$$
\|\nabla p\|_{L^{q}} \leq C_{1}\left\|\left|u\|\nabla u \mid\|_{L^{q}}\right.\right.
$$

holds for any $1<q<\infty$. This relation (2.1) between $\nabla p$ and derivatives of the velocity plays a very important role in the following proof. As far as we know, no one has used (2.1) before.

Multiply both sides of equation (1.1) by $4 u|u|^{2}$, and integrate over $\mathbb{R}^{3}$. After suitable integration by parts, we obtain

$$
\begin{aligned}
& \frac{d}{d t}\|u\|_{L^{4}}^{4}+4\left\|\left|\nabla u\left\|\left.u\left|\left\|_{L^{2}}^{2}+2\right\| \nabla\right| u\right|^{2}\right\|_{L^{2}}^{2}\right.\right. \\
& \quad \leq 4 \int_{\mathbb{R}^{3}}\left|\nabla p\left\|\left.u\right|^{3} d x \leq 4\right\| \nabla p\left\|_{L^{2}}^{1 / 2}\right\| \nabla p\left\|_{L^{\gamma}}^{1 / 2}\right\| u \|_{L^{12 \gamma /(3 \gamma-2)}}^{3}\right. \\
& \quad \leq \quad \epsilon\|\nabla\|_{L^{2}}^{2}+C(\epsilon)\|\nabla p\|_{L^{\gamma}}^{2 / 3}\|u\|_{L^{12 \gamma /(3 \gamma-2)}}^{4} \\
& \quad \leq \quad \epsilon C\left\|\left|\nabla u\|u \mid\|_{L^{2}}^{2}+C(\epsilon)\|\nabla p\|_{L^{\gamma}}^{2 / 3}\|u\|_{L^{4}}^{4(1-1 / \gamma)}\|u\|_{L^{12}}^{4 / \gamma}\right.\right. \\
& \quad \leq \quad \epsilon C\|\mid \nabla u\| u\left\|_{L^{2}}^{2}+C(\epsilon, \delta)\right\| \nabla p\left\|_{L^{\gamma}}^{2 \gamma / 3(\gamma-1)}\right\| u\left\|_{L^{4}}^{4}+\delta\right\| u \|_{L^{12}}^{4},
\end{aligned}
$$

where we used (2.1) for $q=2$. Since

$$
\|u\|_{L^{12}}^{4}=\left\||u|^{2}\right\|_{L^{6}}^{2} \leq C\left\|\left|\nabla u\|u \mid\|_{L^{2}}^{2},\right.\right.
$$

after choosing suitable $\epsilon$ and $\delta$, it follows from (2.2) that

$$
\frac{d}{d t}\|u\|_{L^{4}}^{4} \leq C\|\nabla p\|_{L^{\gamma}}^{2 \gamma / 3(\gamma-1)}\|u\|_{L^{4}}^{4} .
$$

Then applying Gronwall inequality on (2.3), we have

$$
\sup _{0 \leq t \leq T}\|u(., t)\|_{L^{4}}^{4} \leq\left\|u_{0}\right\|_{L^{4}}^{4} \exp \left\{\int_{0}^{T}\|\nabla p(., \tau)\|_{L^{\gamma}}^{2 \gamma / 3(\gamma-1)} d \tau\right\} .
$$

If $1<\alpha, \gamma<\infty$, note that $2 \gamma / 3(\gamma-1) \leq \alpha$. Due to the integrability of $\nabla p$, it follows that

$$
\sup _{0 \leq t \leq T}\|u(., t)\|_{L^{4}}^{4} \leq C(T)\left\|u_{0}\right\|_{L^{4}}^{4} .
$$

For $(\alpha, \gamma)=(2 / 3, \infty)$, by taking the limit case in (2.2), we obtain that

$$
\begin{aligned}
& \frac{d}{d t}\|u\|_{L^{4}}^{4}+\left.4\||| \nabla u\| u\left|\left\|_{L^{2}}^{2}+2\right\| \nabla\right| u\right|^{2} \|_{L^{2}}^{2} \\
& \quad \leq \quad \epsilon C\left\|\left|\nabla u\|u \mid\|_{L^{2}}^{2}+C(\epsilon, \delta)\|\nabla p\|_{L^{\infty}}^{2 / 3}\|u\|_{L^{4}}^{4}+\delta\|u\|_{L^{12}}^{4} .\right.\right.
\end{aligned}
$$

Then by Gronwall inequality, (2.4) follows from (3.9). 
Similarly, for $(\alpha, \gamma)=(\infty, 1)$, we have

$$
\begin{aligned}
& \frac{d}{d t}\|u\|_{L^{4}}^{4}+4\left\|\left|\nabla u\left\|\left.u\left|\left\|_{L^{2}}^{2}+2\right\| \nabla\right| u\right|^{2}\right\|_{L^{2}}^{2}\right.\right. \\
& \quad \leq \quad \epsilon C\left\|\left|\nabla u\left\|u\left|\left\|_{L^{2}}^{2}+C(\epsilon)\right\| \nabla p\left\|_{L^{1}}^{2 / 3}\right\|\right| \nabla u\right\| u\right|\right\|_{L^{2}}^{2} .
\end{aligned}
$$

So if $\sup _{0 \leq t \leq T}\|\nabla p(., t)\|_{L^{1}}$ is sufficiently small, say $\epsilon C \leq 2$ and

$$
C(\epsilon) \sup _{0 \leq t \leq T}\|\nabla p(., t)\|_{L^{1}}^{2 / 3} \leq 2,
$$

then

$$
\sup _{0 \leq t \leq T}\|u(., t)\|_{L^{4}}^{4} \leq\left\|u_{0}\right\|_{L^{4}}^{4} .
$$

In order to prove Theorem 1.1 we recall a result of Giga [6] (see also [9]).

Theorem $2.1\left([\underline{6})\right.$. Suppose $u_{0} \in L^{s}\left(\mathbb{R}^{3}\right), s \geq 3$. Then there exists $T_{0}$ and a unique classical solution $u \in B C\left(\left[0, T_{0}\right) ; L^{s}\left(\mathbb{R}^{3}\right)\right)$. Moreover, let $\left(0, T_{*}\right)$ be the maximal interval such that $u$ solves (1.1) in $C\left(\left(0, T_{*}\right) ; L^{s}\left(\mathbb{R}^{3}\right)\right), s>3$. Then

$$
\|u(., \tau)\|_{L^{s}} \geq \frac{C}{\left(T_{*}-\tau\right)^{(s-3) / 2 s}}
$$

with constant $C$ independent of $T_{*}$ and $s$.

Proof of Theorem 1.1. Since $u_{0} \in L^{2}\left(\mathbb{R}^{3}\right) \cap L^{q}\left(\mathbb{R}^{3}\right)$ for some $q \geq 4$, then $u_{0} \in$ $L^{4}\left(\mathbb{R}^{3}\right)$. Due to Theorem 2.1, there is a maximal interval $\left[0, T_{*}\right)$ such that there exists a unique solution $\tilde{u}(x, t) \in B C\left(\left[0, T_{*}\right) ; L^{4}\left(\mathbb{R}^{3}\right)\right)$. Since $u$ is a Leray-Hopf weak solution which satisfies the energy inequality, we have by the uniqueness criterion of Serrin-Masuda [16, 13]

$$
u \equiv \tilde{u} \quad \text { on } \quad\left[0, T_{*}\right) .
$$

By the a priori estimate, (2.4) or (2.7), and combined with the standard continuation argument, we can continue our local smooth solution corresponding to $u_{0} \in L^{4}\left(\mathbb{R}^{3}\right)$ to obtain $u \in B C\left([0, T] ; L^{4}\left(\mathbb{R}^{3}\right)\right) \cap C^{\infty}\left(\mathbb{R}^{3} \times(0, T]\right)$. This completes the proof of Theorem 1.1.

Remark 2.1. By the same trick as that used in section 3, one can establish an a priori estimate for $\|\nabla p\|_{L^{s}}$ with $3 \leq s<4$.

\section{A NeW PRoOf For Theorem 1.2}

The first step is to give an interpolation inequality.

Lemma 3.1. Suppose a measurable function $f \in L^{\infty, s} \cap L^{s, 3 s}$ on $\left(\mathbb{R}^{3} \times[0, T)\right)$. Then $f \in L^{p, q}$ with $s \leq p, s \leq q \leq 3 s$ and $\frac{s}{p}+\frac{3 s}{2 q} \geq \frac{3}{2}$, and

$$
\|f\|_{L^{p, q}} \leq C(p, q, T)\|f\|_{L^{\infty, s}}^{\frac{3 s-q}{2 q}}\|f\|_{L^{s, 3 s}}^{(3 q-3 s) / 2 q},
$$

where $C(s, p, q, T)$ depends on $s, p, q, T$, and $C(p, q, T)=1$ if $\frac{s}{p}+\frac{3 s}{2 q}=\frac{3}{2}$.

Proof.

$$
\begin{aligned}
\|f\|_{L^{p, q}} & =\left(\int_{0}^{T}\|f(., \tau)\|_{L^{q}}^{p} d \tau\right)^{1 / p} \leq\left(\int_{0}^{T}\|f(., \tau)\|_{L^{s}}^{\theta p}\|f(., \tau)\|_{L^{3 s}}^{(1-\theta) p} d \tau\right)^{1 / p} \\
& \leq C(s, p, q, T)\|f\|_{L^{\infty}, s}^{\theta}\|f\|_{L^{s, 3 s}}^{(1-\theta)}
\end{aligned}
$$


where we use the interpolation theorem

$$
\frac{1}{q}=\frac{\theta}{s}+\frac{1-\theta}{3 s}, \quad s \leq q \leq 3 s,
$$

and Hölder's inequality, provided $(1-\theta) p \leq s$.

From (3.2), $1-\theta=\frac{3 q-3 s}{2 q}$, we obtain $\frac{s}{p}+\frac{3 s}{2 q} \geq \frac{3}{2}$. If $\frac{s}{p}+\frac{3 s}{2 q}=\frac{3}{2}$, which implies $1-\theta=\frac{s}{p}$, then obviously $C(s, p, q, T)=1$.

The idea of the proof of Theorem 1.2 is similar to that of Theorem 1.1. Now the only thing we need is the following a priori estimate.

Theorem 3.2. Let $s \geq 3,1<\alpha<\infty$ and $\frac{3}{2}<\gamma<\infty$ be given. Suppose $u_{0} \in$ $L^{s}\left(\mathbb{R}^{3}\right)$ with $\operatorname{div} u_{0}=0$. Assume $(u, p)$ is a smooth solution of (1.1) in $\mathbb{R}^{3} \times(0, T)$ with $u \in L^{\infty, 2}$ and $\nabla u \in L^{2,2}$. If $p \in L^{\alpha, \gamma}$ with $\frac{2}{\alpha}+\frac{3}{\gamma}=2$, then $u \in L^{\infty, s} \cap L^{s, 3 s}$

$$
\sup _{0 \leq t \leq T}\|u(., t)\|_{L^{s}}^{s} \leq 2^{\left[C\|p\|_{\left.L^{\alpha, \gamma}\right]+1}\left\|u_{0}\right\|_{L^{s}}^{s},\right.}
$$

where $C=C(s, \alpha, \gamma)$.

Proof. In order to prove (3.3) we multiply both sides of equation (1.1) by $s u|u|^{s-2}$, and integrate over $\mathbb{R}^{3} \times(0, t), 0<t \leq T$. After suitable integration by parts, we obtain

$$
\begin{gathered}
\|u(., t)\|_{L^{s}}^{s}+s \int_{0}^{t} \int_{\mathbb{R}^{3}}|\nabla u|^{2}|u|^{s-2} d x d \tau+\frac{4(s-2)}{s}\left\|\nabla|u|^{s / 2}\right\|_{L^{2,2}}^{2} \\
\leq\left. 2(s-2) \int_{0}^{t} \int_{\mathbb{R}^{3}}|p||u|^{s / 2-1}|\nabla| u\right|^{s / 2} \mid d x d \tau+\left\|u_{0}\right\|_{L^{s}}^{s},
\end{gathered}
$$

where we used

$$
\begin{aligned}
-s \int_{0}^{t} \int_{\mathbb{R}^{3}} \nabla p \cdot u|u|^{s-2} d x d \tau & =s(s-2) \int_{0}^{t} \sum_{i, j=1}^{3} \int_{\mathbb{R}^{3}} p \frac{\partial u_{j}}{\partial x_{i}} u_{i} u_{j}|u|^{s-4} d x d \tau \\
& \leq\left. 2(s-2) \int_{0}^{t} \int_{\mathbb{R}^{3}}|p||u|^{s / 2-1}|\nabla| u\right|^{s / 2} \mid d x d \tau .
\end{aligned}
$$

If we use the fact that

$$
\left.\left.|\nabla| u\right|^{s / 2}\left|\leq \frac{s}{2}\right| u\right|^{s / 2-1}|\nabla u|
$$

then (3.4) will be reduced as follows:

$$
\begin{aligned}
\|u(., t)\|_{L^{s}}^{s}+2\left\|\nabla|u|^{s / 2}\right\|_{L^{2,2}}^{2} & \leq\left. 2(s-2) \int_{0}^{t} \int_{\mathbb{R}^{3}}|p||u|^{s / 2-1}|\nabla| u\right|^{s / 2} \mid d x d \tau+\left\|u_{0}\right\|_{L^{s}}^{s} \\
& \equiv A+\left\|u_{0}\right\|_{L^{s}}^{s} .
\end{aligned}
$$

Before going to estimate $A$, we recall the well-known equality given by

$$
-\Delta p=\sum_{i, j=1}^{3} \partial_{i} \partial_{j}\left(u_{i} u_{j}\right) .
$$

The Calderon-Zygmund inequality implies

$$
\|p\|_{L^{\gamma}} \leq C_{1}\|u\|_{L^{2 \gamma}}^{2}, \quad 1<\gamma<\infty .
$$


Now,

$$
\begin{aligned}
A \leq & C_{2} \int_{0}^{t}\|p\|_{L^{a}}\|u\|_{L^{b}}^{s / 2-1}\left\|\nabla|u|^{s / 2}\right\|_{L^{2}} d \tau \\
& \left(\text { Hölder's inequality } \frac{1}{a}+\frac{s / 2-1}{b}=\frac{1}{2}\right) \\
\leq & \frac{1}{2} C_{2} \int_{0}^{t}\|p\|_{L^{a}}^{2}\|u\|_{L^{b}}^{s-2} d \tau+\int_{0}^{t}\left\|\nabla|u|^{s / 2}\right\|_{L^{2}}^{2} d \tau \quad \text { (Young's inequality) } \\
\leq & \frac{1}{2} C_{2} \int_{0}^{t}\|p\|_{L^{\gamma}}^{2(1-\theta)}\|p\|_{L^{b / 2}}^{2 \theta}\|u\|_{L^{b}}^{s-2} d \tau+\int_{0}^{t}\left\|\nabla|u|^{s / 2}\right\|_{L^{2}}^{2} d \tau \\
& \left(\text { interpolation inequality } \frac{1}{a}=\frac{1-\theta}{\gamma}+\frac{\theta}{b / 2}\right) \\
\leq & \left.C_{3} \int_{0}^{t}\|p\|_{L^{\gamma}}^{2(1-\theta)}\|u\|_{L^{b}}^{4 \theta+s-2} d \tau+\int_{0}^{t}\left\|\nabla|u|^{s / 2}\right\|_{L^{2}}^{2} d \tau \quad \text { (by } \quad \text { (3.7) }\right) \\
\leq & C_{3}\|p\|_{L^{\alpha, \gamma}}^{2(1-\theta)}\|u\|_{L^{q, b}}^{4 \theta+s-2}+\int_{0}^{t}\left\|\nabla|u|^{s / 2}\right\|_{L^{2}}^{2} d \tau \\
& \left(\text { Hölder's inequality } \frac{2(1-\theta)}{\alpha}+\frac{4 \theta+s-2}{q}=1\right) .
\end{aligned}
$$

We can choose the number $\theta=\frac{1}{2}$; then

$$
a=\frac{2 \gamma s}{2 \gamma+s-2}, \quad b=\frac{\gamma s}{\gamma-1}, \quad q=\frac{\alpha s}{\alpha-1} .
$$

From (3.8), by direct computation, $q$ and $b$ satisfy

$$
\frac{s}{q}+\frac{3 s}{2 b}=\frac{5}{2}\left(\frac{1}{\alpha}+\frac{3}{2 \gamma}\right) \geq \frac{3}{2}, \quad s<q, \quad s<b<3 s,
$$

so we can use inequality (3.1). Therefore

$$
\begin{aligned}
A & \leq C_{3}\|p\|_{L^{\alpha, \gamma}}\|u\|_{L^{q, b}}^{s}+\int_{0}^{t}\left\|\nabla|u|^{s / 2}\right\|_{L^{2}}^{2} d \tau \\
& \leq C_{4}\|p\|_{L^{\alpha, \gamma}}\|u\|_{L^{\infty, s}}^{\frac{2 \gamma-3}{2 \gamma}}\|u\|_{L^{s, 3 s}}^{\frac{3}{2 \gamma} s}+\int_{0}^{t}\left\|\nabla|u|^{s / 2}\right\|_{L^{2}}^{2} d \tau \\
& \leq C_{5}\|p\|_{L^{\alpha, \gamma}}^{\frac{2 \gamma}{2 \gamma-3}}\|u\|_{L^{\infty, s}}^{s}+C_{6}\|u\|_{L^{s, 3 s}}^{s}+\int_{0}^{t}\left\|\nabla|u|^{s / 2}\right\|_{L^{2}}^{2} d \tau,
\end{aligned}
$$

where $C_{5}$ is constant depending only on $\alpha, \gamma$ and $s$, while $C_{6}$ is an absolute constant to be determined later. Substituting the above inequalities into (3.5) and using the Sobolev inequality for suitable $C_{6}$,

$$
C_{6}\|u\|_{L^{3 s}}^{s}=C_{6}\left\||u|^{s / 2}\right\|_{L^{6}}^{2} \leq\left\|\nabla|u|^{s / 2}\right\|_{L^{2}}^{2},
$$

one has

$$
\|u(., t)\|_{L^{s}}^{s} \leq C_{5}\|p\|_{L^{\alpha, \gamma}}^{\frac{2 \gamma}{2 \gamma-3}}\|u\|_{L^{\infty, s}}^{s}+\left\|u_{0}\right\|_{L^{s}}^{s} .
$$

Theorem 3.1 follows from (3.9) and the integrability of $p$.

Remark 3.1. From the proof of Theorem 1.2, it is obvious that Theorem 1.2 holds for arbitrary dimension $N, N \geq 3$. 


\section{ACKNOWLEDGMENT}

The author would like to express sincere gratitude to his supervisor Professor Zhouping Xin for enthusiastic guidance and constant encouragement. The author thanks Professor Berselli for the comments on the first version of this paper and for his interest. Thanks also to the referee for his/her constructive comments. This work was partially supported by Hong Kong RGC Earmarked Grants CUHK-427900P and Shanghai Rising-Star Program 05QMX1417.

\section{REFERENCES}

[1] H. Beirao da Veiga, On the smoothness of a class of weak solutions to the Navier-Stokes equations. J. Math. Fluid Mech., 2(2000), no. 4, 315-323. MR1814220 (2001m:35250)

[2] L.C. Berselli, G.P. Galdi, Regularity criteria involving the pressure for the weak solutions to the Navier-Stokes equations, Proc. Amer. Math. Soc., 130(2002), no. 12, 3585-3595 (electronic). MR1920038 (2003e:35240)

[3] L. Caffarelli, R. Kohn, L. Nirenberg, Partial regularity of suitable weak solutions of the Navier-Stokes equations, Comm. Pure Appl. Math., 35(1982), 771-831. MR0673830 (84m:35097)

[4] D. Chae, J. Lee, Regularity criterion in terms of pressure for the Navier-Stokes equations, Nonlinear Analysis, 46(2001), 727-735. MR1857154 (2002g:76032)

[5] P. Constantin, C. Foias, Navier-Stokes equations, Chicago Lectures in Mathematics series, (1988). MR0972259 (90b:35190)

[6] Y. Giga, Solutions for semilinear parabolic equations in $L^{p}$ and regularity of weak solutions of the Navier-Stokes system, J. Differential Equations, 62(1986), 186-212. MR0833416 (87h:35157)

[7] E. Hopf, Uber die Anfangswertaufgabe fur die hydrodynamischen Grundgleichungen (German), Math. Nachr., 4(1951), 213-231. MR0050423(14:327b)

[8] L. Iskauriaza, G. A. Seregin, V. Shverak, $L_{3, \infty}$-solutions of Navier-Stokes equations and backward uniqueness (Russian), Uspekhi Mat. Nauk, 58(2003), no. 2, 3-44. MR1992563 (2004m:35204)

[9] T. Kato, Strong $L^{p}$-solutions to the Navier-Stokes equations in $\mathbb{R}^{m}$, with applications to weak solutions, Math. Z., 187(1984), 471-480. MR0760047 (86b:35171)

[10] H. Kozono, H. Sohr, Regularity criterion on weak solutions to the Navier-Stokes equations, Adv. Differential Equations, 2(1997), 535-554. MR1441855 (97m:35206)

[11] H. Kozono, Y. Taniuchi, Bilinear estimates in $B M O$ and the Navier-Stokes equations, Math. Z., 235(2000), 173-194. MR1785078 (2001g:76011)

[12] J. Leray, Étude de divers équations intégrales nonlinearies et de quelques problemes que posent lhydrodinamique, J. Math. Pures. Appl., 12(1931), 1-82.

[13] K. Masuda, Weak solutions of the Navier-Stokes equations, Tohoku Math. J., 36(1984), 623-646. MR0767409 (86a:35117)

[14] M. O'Leary, Pressure conditions for the local regularity of solutions of the Navier-Stokes equations, Electron. J. Differential Equations (12)(1998), 1-9. MR1625358 (99c:35188)

[15] V. Scheffer, Partial regularity of solutions to the Navier-Stokes equations, Pacific J. Math., 66(1976), 535-552. MR0454426 (56:12677)

[16] J. Serrin, On the interior regularity of weak solutions of the Navier-Stokes equations, Arch. Rational Mech. Anal., 9(1962), 187-195. MR0136885 (25:346)

[17] H. Sohr, Zur Regularitatstheorie der instationaren Gleichungen von Navier-Stokes. Math. Z. 184(1983), no. 3, 359-375. MR0716283(85f:35167)

[18] M. Struwe, On partial regularity results for the Navier-Stokes equations, Comm. Pure Appl. Math., 41(1988), 437-458. MR0933230 (89h:35270)

[19] R. Temam, Navier-Stokes equations, theory and numerical analysis, AMS Chelsea Publishing (2001). MR 1846644 (2002j:76001)

[20] G. Tian, Z. Xin, Gradient Estimation on Navier-Stokes equations, Comm. Anal. Geo., 7 (1999), 221-257. MR1685610 (2000i:35166) 
[21] W. von Wahl, Regularity of weak solutions of the Navier-Stokes equations, Proceedings of the 1983 Summer Institute on Nonlinear Functional Analysis and Applications, Proc. Symposia in Pure Mathematics 45, Amer. Math. Soc., Providence, Rhode Island, (1986), 497-503. MR0843635 (87g:35193)

[22] Y. Zhou, Regularity criteria in terms of pressure for the 3-D Navier-Stokes equations in a generic domain, Math. Ann., 328(2004), no. 1-2, 173-192. MR2030374 (2004j:35229)

Department of Mathematics, East China Normal University, Shanghai, 200062, PeoPLE'S REPUBLIC OF CHINA

E-mail address: yzhou@math.ecnu.edu.cn 